\title{
Uso de quitina y quitosana procedentes de residuos de camarón en la purificación de jugos de caña (saccharum officinarum)
}

\author{
DURÁN-OLVERA, JESSY MARLEN', MAGAÑA-PÉREZ, GUADALUPE' , AYALA-TIRADO ROSA CARMINA², GARCÍA-GÓMEZ, ROLANDO S. \\ AMÁBILIS-SOSA, LEONEL ERNESTO ${ }^{4}$, DURÁN-DE-BAZÚA, MARÍA DEL CARMEN³ y SOLÍS-FUENTES, JULIO ALBERTO2* \\ 11) Facultad de Ciencias Químicas, Zona Xalapa, U. V. \\ (2) Instituto de Ciencias Básicas, Universidad Veracruzana. \\ Av. Dos Vistas s/n carretera Xalapa-Las Trancas, 91000 Xalapa, Ver., México \\ Fax (+52) -2841-8931. \\ (3) Facultad de Química, UNAM \\ Conjunto "E", Labs 301-303, Ciudad Universitaria, 04510 México, D.F., México \\ Fax (+52) 55-5622-5303. Correo-e (e-maill:mcduran@unam.mx \\ (4) Universidad Politécnica de la Energía, Tula, Hidalgo, México. Correo-e: leoamabilis@yahoo.com.mx \\ *Correspondencia: jsolis@uv.mx,jsolisjulio@gmail.com
}

Recibido: 28 de enero de 2015, Aceptado: 9 de agosto de 2016

\section{Resumen}

En este trabajo se estudió la clarificación de jugo de caña (guarapo' y jugo claro) empleando quitina y quitosana obtenidas de exoesqueletos y cefalotórax de camarón como agentes precipitantes. La obtención de la quitina se realizó mediante desproteinización y desmineralización química de los exoesqueletos y la quitosana por desacetilación de la quitina. Ambos biopolímeros se analizaron mediante espectroscopía infrarroja IR y se caracterizaron fisicoquímicamente. Los resultados mostraron que el guarapo y el jugo clarificado tuvieron originalmente un color de 35333 y $19938 \mathrm{UI}$, respectivamente. Los tratamientos con quitina y con quitosana lograron remover hasta el 49.9 y $69.61 \%$, respectivamente del color original del jugo de primera extracción y hasta el 56.37 y $74.22 \%$, respectivamente, del color original del jugo claro de caña en tratamientos realizados a un $\mathrm{pH}$ de 3.0 y temperatura ambiente. Ambos materiales mostraron potencialidad como opciones de purificación del jugo de caña de azúcar.

Palabras clave: clarificación, quitina, quitosana, residuos de camarón

\section{Use of chitin and chitosan from shrimp wastes in the purification of sugar cane (saccharum officinarum) juices}

\begin{abstract}
In this research the clarification of sugarcane juice (guarapo and clarified juice) with both chitin and chitosan obtained from shrimp exoskeletons and cephalothorax was studied. Chitin was produced by chemical demineralisation and deproteinization of the shrimp residues and chitosan through chitin deacetylation. Both biopolymers were analyzed by IR spectroscopy and physicochemically characterized. The results show that guarapo and clarified cane juice had 35333 and $19938 \mathrm{IU}$ of color, respectively, and that chitin and chitosan were able to remove till $49.9 \%$ and $69.61 \%$, respectively of the color of the first extraction cane juice; and $56.37 \%$ and $74.22 \%$, respectively of the original color of the clarified cane juice through treatments realized at $\mathrm{pH} 3.0$ and ambience temperature. Both materials showed potentiality as options for cane juice purification.
\end{abstract}

Keywords: clarification, chitin, chitosan, shrimp waste

1 Guarapo: Voz quechua. 1. m. Am. Jugo de la caña dulce exprimida que por vaporización produce el azúcar (RAE, 2015) 


\section{Introducción}

La agroindustria de la caña de azúcar es una de las de mayor importancia en varios países de América Latina, Asia y África. En México, esta agroindustria ha hecho propicio, desde las primeras instalaciones trapicheras ubicadas en lo que hoy es el estado de Veracruz, un desarrollo y especialidad regional que hoy en día es sostén económico y social de millones de personas que directa $\circ$ indirectamente se relacionan con las actividades productivas y comerciales del sector azucarero mexicano (Enríquez-Poy, 2015).

Es en función de esa destacada importancia social y económica el que la compleja problemática económica, comercial, financiera y tecnológica que enfrenta esta agroindustria en el contexto de la globalidad actual sea prioritaria y atendida por los diversos actores gubernamentales, empresariales y académicos para hallar las soluciones apropiadas y requeridas. En ese conjunto de aspectos, la innovación tecnológica en sus plantas de procesamiento y la diversificación de su producción juegan un papel de gran relevancia (Aguilar-Rivera y col., 2009; Domínguez, 2005).

La producción de azúcar de caña por lo general involucra varias y distintas operaciones que incluyen un buen número de tratamientos físicos y químicos. El proceso generalizado de fabricación de azúcar de caña utilizado en la industria comprende numerosos pasos de purificación de las soluciones que contienen la sacarosa que en su origen es el jugo de caña. En tales pasos, las impurezas presentes en el material de inicio se eliminan con el fin de obtener finalmente la sacarosa lo más pura posible (Clarke y Godshall, 2013).

Gran parte de las innovaciones sugeridas e investigadas más ampliamente se refieren a métodos alternativos para la purificación y decoloración del jugo (Clarke y Roberts, 1996; Hinkova y col., 2002; Honig, 2013; Paananen y Wen, 1999; Qudsieh y col.,
2002; Šereš y col., 2006). Esto es porque estas operaciones determinan en un alto porcentaje la pureza y rendimientos de la sacarosa.

En esta investigación se estudió la clarificación de jugo de caña con quitina y con quitosana obtenidas de residuos de camarón, abundantes en México. Considerando que el valor de la producción de camarón en el 2013 fue de 7,521 millones de pesos (con un equivalente aproximado de 17 pesos mexicanos por dólar estadounidense), con una producción de 127.5 miles de toneladas, esta industria es muy importante para México (Flores-Ortega y col., 2004; Ortega-Granados y Durán-Domínguez-de-Bazúa, 2014; TéllezCastañeda, 2014). Desde el inicio de su industrialización en granjas, además de su captura en el mar, se han ido acumulando una gran cantidad de residuos procedentes de esas actividades alcanzando en México una producción estimada de aproximadamente 60 mil toneladas anuales. Como muchas otras industrias, las procesadoras de los productos de la pesca y, particularmente del camarón, desechan con frecuencia sus residuos a los cuerpos de agua existentes en su entorno, provocando impactos ambientales importantes por su lenta degradación (Beléndez-Moreno y col., 2014). La Figura 1 muestra una fotografía publicada en una tesis de posgrado de México (Ortega-Granados, 2014) sobre esta problemática.

Los exoesqueletos y cefalotórax de crustáceos y, particularmente, los de camarón son la principal fuente de quitina. Este compuesto está ampliamente distribuido en la naturaleza y es después de la celulosa el polímero natural más abundante. Presenta una tasa de reposición tan alta en la biosfera que -se estimaduplica a la de la celulosa y es a partir de él que se obtiene la quitosana a través de diferentes procesos fisico-químicos y/o enzimáticos (Flores-Ortega, 2004, 2008, Flores Ortega y col., 2004). 


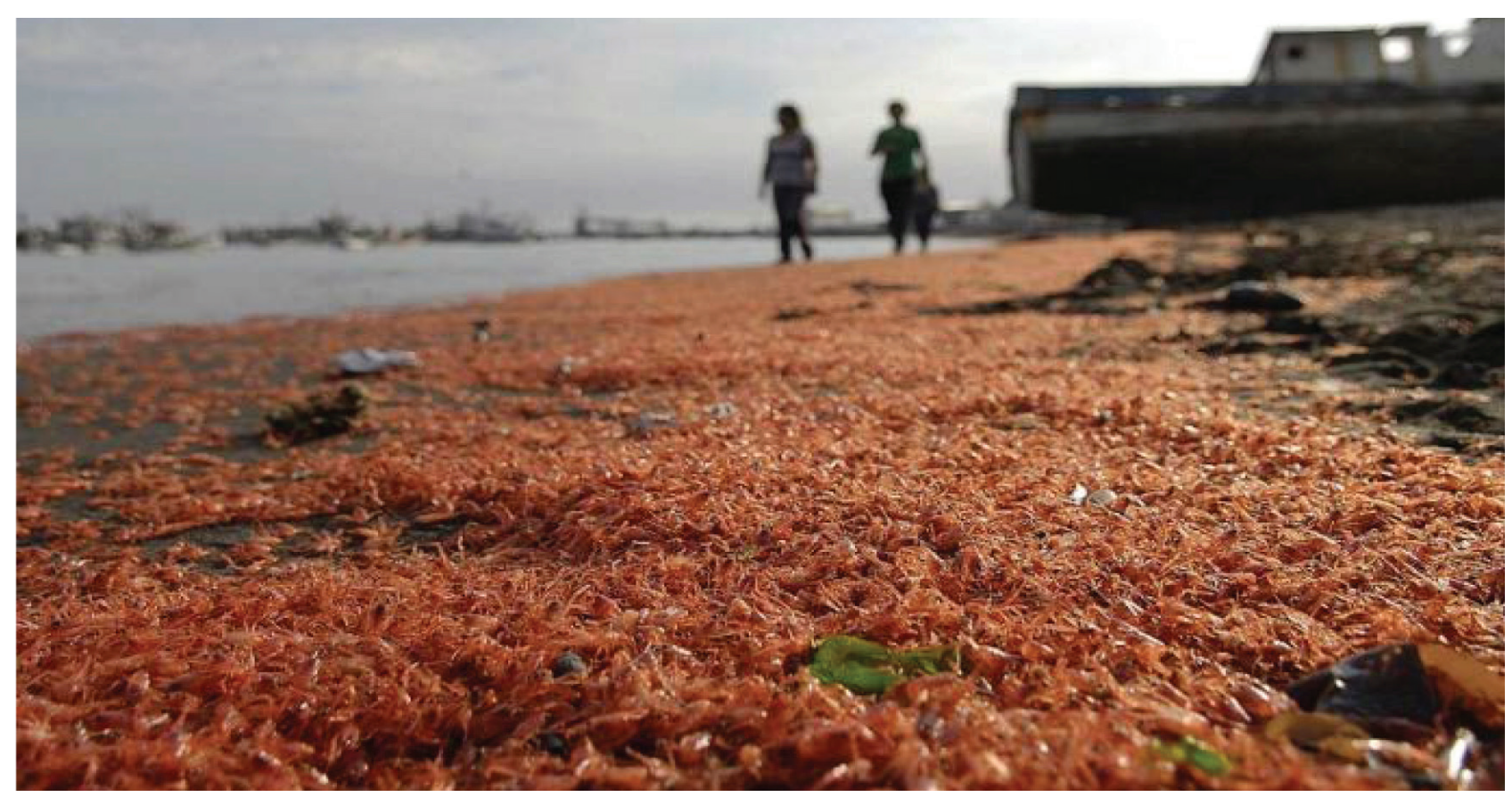

Figura 1. Fotografía que muestra los residuos de una empresa empacadora de camarón contaminando el entorno, en este caso en la orilla del mar (Ortega-Granados, 2014)

La quitina es un polisacárido (poli N-acetil-glucosamina) que mediante reacciones de parcial desacetilación se convierte en quitosana poli ( $\mathrm{N}$-acetil-glucosamina y $\beta$-(1-4) D- glucosamina) o mediante total desacetilación, en quitano (Lárez-Velásquez, 2006). Es interesante mencionar que este autor denomina quitosana al polímero que tiene un $50 \%$ de desacetilación y quitana al que está $100 \%$ desacetilado, usando los términos en género masculino (quitosano y quitano, respectivamente) aunque las gomas, según la química tradicional, tienen una terminación femenina (dextrana, pululana, etc.).

La quitina y la quitosana son materiales de amplio uso en diversos campos de aplicación como: la agricultura, medicina, tratamiento de aguas, cosméticos, alimentos, entre otros (Lárez-Velásquez, 2006; Mármol y col., 2011$)$.

En el interés de la presente investigación destacan sus usos como agentes floculantes, coagulantes y removedores de colorantes en procesos de tratamiento de aguas y bebidas de frutas, mostrando con ello ser materiales con características comestibles y ambientalmente amigables a la par de ser importantes en las vías de reciclamiento para los abundantes residuos de los productos marinos de donde provienen.
La quitina ha sido evaluada como adsorbente de compuestos cromóforos y fenólicos en vinos (Mármol y col., 2009) y la quitosana es ampliamente considerada como un buen agente coagulante y clarificante en el tratamiento de jugos de frutas y de aguas residuales teniendo su uso referencias clásicas como las de Bough y colaboradores (Bough, 1975; Bough y Landes, 1978; Oszmiański y Wojdyło, 2007) y más recientes como las de Castro-Domingues y colaboradores (2011) y de Tastan y Baysal (2015).

En este trabajo se evaluó la clarificación del jugo de caña con quitina y quitosana obtenidos de residuos de camarón. Esto en el marco de un proyecto general que investiga la obtención de adsorbentes a partir de residuos biológicos y agroindustriales para su aplicación en procesos de purificación en la industria azucarera. Se planteó la evaluación de estos dos importantes materiales, provenientes de un residuo biológico abundante en México, las cabezas (cefalotórax) y las cáscaras (exoesqueletos) de los camarones, en la remoción de materiales que están presentes en los jugos de caña que se procesan en la industria azucarera para la fabricación de sacarosa y le confieren las propiedades indeseables de color y turbidez, especialmente para los diversos propósitos de procesamiento del jugo, sea la obtención de sacarosa cristalina, la producción de azúcares líquidos o la posibilidad de obtener diversos jarabes hidrolizados o no. 


\section{Materiales y métodos}

\section{Diseño experimental y análisis estadís- ticos}

La presente investigación se basa en evaluar el porcentaje de remoción de color del guarapo y jugo claro (ambos del jugo de caña), a través de quitina y quitosana y su combinación a tres valores de $\mathrm{pH}$. Dado que cualquier combinación es posible y contrastada contra las demás, se conforma un experimento de bloques aleatorios para comparaciones completamente al azar (Montgomery, 2011). A pesar de que las condiciones del diseño experimental sugieren un análisis de varianza (ANDEVA), este sólo aplica cuando el experimento es de tipo paramétrico, lo cual se suscita por la combinación de tener un alto número de datos, un número de réplicas que produzcan mayores grados de libertad y una distribución que obedezca a la función de normalidad (gaussiana). En este orden de ideas, se tiene la restricción de no contar con réplicas, dada la dificultad de obtener la materia prima y productos del laboratorio; además de la necesidad de utilizar la misma proporción de muestras con sus réplicas para cada valor de $\mathrm{pH}$ que fue evaluado (se requirieron cuatro muestras de quitosana y cuatro de quitina para poder variar el $\mathrm{pH}$ ). Esta restricción logística, sugiere que la distribución de los datos no es paramétrica, por lo cual no se puede utilizar el análisis ANDEVA. Afortunadamente, el diseño de bloques al azar, también permite que los datos puedan ser anali- zados por pruebas no paramétricas y con ello obtener resultados con fundamentos estadísticos (Montgomery, $2011)$. En el caso particular de este experimento, el efecto del $\mathrm{pH}$ y el tipo de sustrato clarificante (quitina y quitosana) puede ser evaluado por medio de la remoción de color, utilizando el análisis Kruskall-Wallis. Con base en todo lo anterior, las diferencias significativas para cada comparación fueron analizadas con dicho análisis estadístico, a través de la paquetería Statgraphics Centurion XV:II, garantizando la fiabilidad de las comparaciones. En ellas, para cada tipo de jugo (dos factores: guarapo y jugo claro) se aplicó la combinación de dos factores: factor uno compuesto clarificante con dos niveles, quitina y quitosana y factor dos $\mathrm{pH}$ con cuatro niveles, 3, 4.3, 7 y 11 . La combinación arroja 16 tratamientos diferentes como se muestra en la Tabla 1.

La realización de este trabajo incluyó la obtención y caracterización fisicoquímica de muestras de jugos de caña (de primera extracción o guarapo y de jugo claro) de un ingenio azucarero. La obtención de desechos del camarón como fuente para la elaboración de quitina y quitosana, la caracterización de estos compuestos obtenidos en laboratorio, la remoción de color en los jugos de caña y la valoración de la quitina y la quitosana como adsorbente y agente coagulofloculante en la remoción de compuestos cromóforos del jugo. La Figura 2 muestra en un diagrama la ruta metodológica general seguida en este trabajo.

Tabla 1. Diseño experimental y análisis estadístico

\begin{tabular}{|c|c|c|}
\hline Jugo & Compuesto clarificante & pH \\
\hline Guarapo & Quitina & 3.0 \\
\hline Guarapo & Quitina & 4.3 \\
\hline Guarapo & Quitina & 7.0 \\
\hline Guarapo & Quitina & 11 \\
\hline Guarapo & Quitosana & 3.0 \\
\hline Guarapo & Quitosana & 4.3 \\
\hline Guarapo & Quitosana & 7.0 \\
\hline Guarapo & Quitosana & 11.0 \\
\hline Jugo claro & Quitina & 3.0 \\
\hline Jugo claro & Quitina & 4.3 \\
\hline Jugo claro & Quitina & 7.0 \\
\hline Jugo claro & Quitina & 11.0 \\
\hline Jugo claro & Quitosana & 3.0 \\
\hline Jugo claro & Quitosana & 4.3 \\
\hline Jugo claro & Quitosana & 7.0 \\
\hline Jugo claro & Quitosana & 11.0 \\
\hline
\end{tabular}




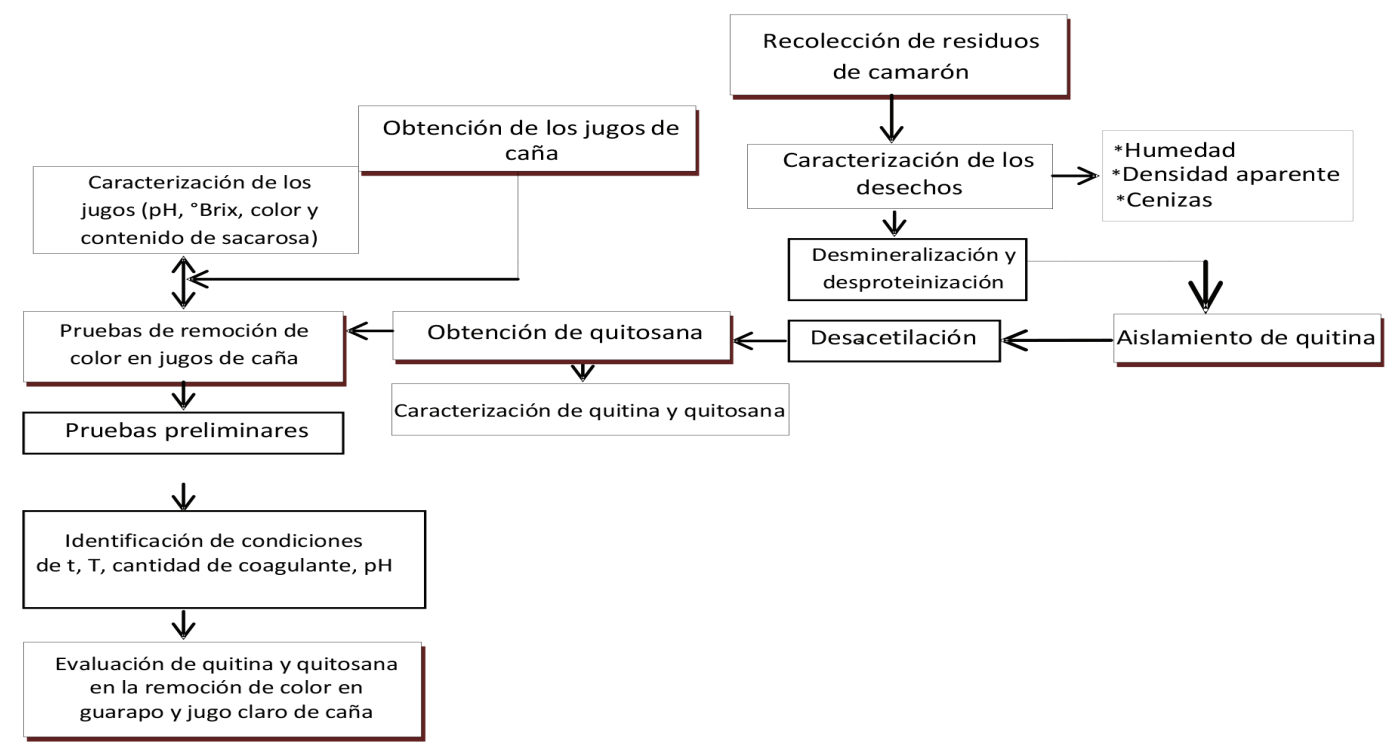

Figura 2. Diagrama de la metodología utilizada

\section{Obtención y caracterización de las muestras de jugos de caña}

Las muestras de los jugos, de primera extracción o guarapo y jugo claro, fueron obtenidas del ingenio El Modelo S. A. de C. V., ubicado en el Municipio de José Cardel en el Estado de Veracruz, México. Las muestras fueron transportadas al laboratorio, equilibradas térmicamente y refrigeradas a temperaturas de $-10^{\circ} \mathrm{C}$ hasta su utilización.

\section{Acondicionamiento y caracterización fi- sicoquímica de los jugos de caña}

Para efectuar las determinaciones el jugo claro fue filtrado y el guarapo (jugo de primera extracción) centrifugado a $4000 \mathrm{rpm}$ durante $20 \mathrm{~min}$ y posteriormente filtrado. Las muestras de jugo fueron analizadas en términos de sus principales características fisicoquímicas: ${ }^{\circ}$ Brix, pH, contenido de sacarosa y color, utilizando las técnicas oficiales (ICUMSA, 2006).

\section{Determinación de sólidos totales}

Para esta valoración se tomaron muestras de $5 \mathrm{~g}$ de jugo de caña y se colocaron en charolas previamente secas y a masa constante, éstas fueron colocadas en una estufa a temperatura de $100-110^{\circ} \mathrm{C}$, con registros de masa cada hora y periodos de enfriamiento en el desecador de $5 \mathrm{~min}$, hasta alcanzar masa constante.
La densidad se determinó mediante la medida de la masa, en gramos, de $25 \mathrm{~mL}$ de una muestra de jugo de caña y la utilización de la expresión $d=m / v$. Los sólidos solubles se determinaron usando un refractómetro digital marca Sper Scientific modelo 300035 y el valor de $\mathrm{pH}$ con un potenciómetro marca Oakton modelo WD-3561872 pH 310. Para esto último $25 \mathrm{~mL}$ de cada muestra en agitación moderada fueron puestos en contacto con el electrodo del potenciómetro hasta la estabilización de la lectura resultante.

\section{Color ICUMSA en los jugos de caña}

En esta determinación se empleó un espectrofotómetro marca Thermoscientific Genesys 10 S UV-Vis. en base a la absorbancia y ${ }^{\circ}$ Brix se realizó una dilución de las muestras de modo que las lecturas de transmitancia estuvieran entre 20 y $80 \%$ a $420 \mathrm{~nm}$. Se ajustó el pH a $7.0 \pm 0.02$ unidades utilizando soluciones de $\mathrm{NaOH}$ y $\mathrm{HCl}$ de 1 y $0.1 \mathrm{~mol} / \mathrm{L}$ respectivamente y se procedió con la medición de la absorbancia de la solución, utilizando agua destilada como referencia. El color, en unidades de color ICUMSA (ICUMSA, 2006), se determinó, usando la expresión:

$\operatorname{Color}(\boldsymbol{U I})=\frac{A_{s}}{b c}(1000)$ 
donde: $A_{s}=$ absorbancia; $b=$ longitud de la celda (en $\mathrm{cm}) ; c=$ concentración de sólidos totales (en $\mathrm{g} / \mathrm{mL})$ determinada por refractómetro y calculada a partir de la densidad y $1000=$ factor convencional.

\section{Pol' y contenido de sacarosa en los jugos de caña}

Se utilizó un polarímetro marca Polax-2L, para medir el contenido de sacarosa presente en los jugos. Se tomó una muestra de $50 \mathrm{~mL}$ de jugo o guarapo, a la cual se añadió $2 \mathrm{~g}$ de acetato de plomo con agitación constante a $300 \mathrm{rpm}$ durante $45 \mathrm{~min}$, con el fin de obtener una solución clarificada, previo a la lectura en el polarímetro. Se utilizó agua desionizada para la calibración. El pol registrado se utilizó para estimar el contenido de sacarosa de la muestra (Chen, 1991).

\section{Obłención y acondicionamiento de las muestras de residuos de camarón}

Se obtuvieron muestras de exoesqueletos de camarón mediante la recolección de desechos de camarón en diferentes establecimientos de comida y restaurantes de la ciudad de Xalapa, Ver. Los caparazones (exoesqueletos) fueron separados de los demás constituyentes de los residuos del crustáceo (como patas, cabezas y colas), para luego ser lavados cuidadosamente con agua. Los exoesqueletos limpios fueron secados en una estufa a temperatura entre 60 y $70^{\circ} \mathrm{C}$ hasta masa constante, para después ser molidos y tamizados, buscando obtener tamaños de partícula menores a 250 $\mu \mathrm{m}$. El material pulverizado así obtenido se almacenó en frascos de plástico y sellados, hasta su utilización.

\section{Caracterización de los cefalotórax y exoesqueletos de camarón}

El material pulverizado recuperado de los exoesqueletos de camarón fue sometido a determinaciones fisicoquímicas de porcentaje de cenizas, humedad y densidad aparente de acuerdo con las técnicas oficiales de análisis AOAC (Horwitzs, 1995).

\section{Aislamiento químico de quitina de exoesqueletos y cefalotórax de cama- rón}

La obtención de quitina se llevó a cabo mediante métodos químicos de desmineralización y desproteiniza-

1 Pol es un término muy usado en la industria azucarera para definir la concentración de sacarosa aparente empleando el método de polarización de un producto azucarado y aforado con agua a $100 \mathrm{~mL}$ a 293K preclarificado con subacetato de plomo seco de Horne en un tubo polarimétrico de $200 \mathrm{~mm}$ de longitud usando un sacarímetro con escala internacional en grados S (DOF, 1991, Norma Mexicana NMXF-271-1991, COLPOS, 1991) ción en base a la metodología descrita por Tolaimate y colaboradores (2000). Brevemente y de acuerdo con esta referencia, se pesó una cantidad de exoesqueletos de camarón pulverizado y previamente tamizado, con tamaño de partícula menor a 250 pm. El material fue colocado en un vaso de precipitado conteniendo una solución de $\mathrm{HCl} 0.6 \mathrm{~N}$ en una relación 1:11 (sólido-líquido) a temperatura de $30^{\circ} \mathrm{C}$ durante 3 horas. Al cabo de ese tiempo la muestra se lavó con agua destilada hasta la eliminación total del ácido. La desproteinización de la muestra utilizó una solución de $\mathrm{NaOH}$ al $1 \%$ a una temperatura de $28^{\circ} \mathrm{C}$ durante 24 horas de agitación constante para asegurar completa desproteinización midiendo nitrógeno Kjeldahl y determinando que quedara solamente nitrógeno no proteico (Cañipa-Morales, 1994). Al término de dicho tratamiento, la muestra se lavó nuevamente con agua destilada hasta alcanzar la neutralidad. El material sólido resultante es la quitina aislada.

\section{Obtención química de la quitosana}

La quitina obtenida, se sometió a un proceso de desacetilación, mediante el cual fue convertida en quitosana. Para ello, se pesó una cantidad de la quitina previamente aislada y se vertió en una solución de $\mathrm{NaOH}$ al $50 \%$ en una relación 1:4 (sólido- líquido), manteniéndose las siguientes condiciones: 2 horas a $60^{\circ} \mathrm{C}$ y luego 2 horas a $100^{\circ} \mathrm{C}$. Posteriormente la muestra se lavó con agua destilada hasta $\mathrm{pH}$ neutro (Tolaimate y col., 2000). El producto así obtenido es la quitosana.

\section{Caracterización fisicoquímica de la qui- tina y la quitosana obtenidas}

Ambos materiales fueron sometidos a una serie de pruebas fisicoquímicas con la finalidad de determinar primero, si efectivamente se llegó a su obtención; y segundo, para determinar algunas de sus características fisicoquímicas. Para ello se usó la espectroscopía infrarroja y técnicas oficiales de análisis para: porcentaje de materia insoluble, pérdida por desecación o sólidos totales, cenizas o residuos de ignición, y densidad aparente, y grado de desacetilación (GD) sólo a la quitosana.

\section{Identificación mediante espectroscopía infrarroja (IR)}

Los espectros IR de de la quitina y la quitosana fueron obtenidos en un espectrofotómetro de infrarrojo marca Beckman Acculab 4 en la región de $800-4000 \mathrm{~cm}^{-1}$. Se elaboraron pastillas con $100 \mathrm{mg}$ de bromuro de pota- 
sio por cada mg de muestra (Mc Kay y col., 1987). Se utilizó quitina comercial también en este análisis, de la marca Sigma, para efectos de comparación de los espectros resultantes.

\section{Grado de desacetilación (GD) de la quitosana mediante valoración potenciométrica}

Para la determinación de grupos aminos de la muestra de quitosana se procedió a la disolución de $0.5 \mathrm{~g}$ de este material en $20 \mathrm{~mL}$ de $\mathrm{HCl}$ 0.3M. Después, se tituló con una solución de $\mathrm{NaOH}$ 0.1M. La valoración se llevó a cabo mediante la medición del cambio de $\mathrm{pH}$ cada $2 \mathrm{~mL}$ de base añadida, la adición se realizó en forma lenta y con agitación continua. Se obtuvo la curva de titulación con dos puntos de inflexión. El cálculo se realizó utilizando la expresión:

$$
\% \mathrm{NH}_{2}=\frac{16.1(x-y)}{w} x f
$$

donde: $y=$ punto de inflexión mayor; $x=$ punto de inflexión menor expresado en volumen; $f$ = molaridad de la solución de $\mathrm{NaOH} ; \mathrm{w}=$ masa en gramos de la muestra y 16.1 es un factor asociado con la masa equivalente de quitosana (Hidalgo y col., 2009).

\section{Porcentaje de materia insoluble de la quitosana}

Se determinó disolviendo la quitosana al $0.5 \%(\mathrm{p} / \mathrm{v})$ en una solución de ácido acético $0.1 \mathrm{M}$ con agitación constante a $250 \mathrm{rpm}$ durante $24 \mathrm{~h}$; después, la muestra se filtró en papel filtro y se secó en una estufa a temperatura de $105-110^{\circ} \mathrm{C}$ hasta masa constante. Los cálculos se realizaron con base en la diferencia de masa (Goycoolea y col., 2000).

\section{Contenido de cenizas}

$1 \mathrm{~g}$ del material de ensayo fue incinerada en una parrilla eléctrica hasta total carbonización y desaparición de humos blancos. Posteriormente se incineró en mufla a $750-800^{\circ} \mathrm{C}$, durante 6 horas. Se enfrió el crisol en un desecador hasta temperatura ambiente y se pesó, la muestra fue regresada a la mufla hasta masa constante. La prueba fue realizada por duplicado, y los resultados fueron obtenidos en base a las diferencias de masa.

\section{Efecto de la cantidad de agente coagulante}

En esta valoración, $60 \mathrm{~mL}$ de jugo fueron puestos en contacto con el adsorbente a diferentes cantidades bajo las siguientes condiciones: agitación de 200 rpm (para evitar la ruptura de los flóculos) y temperatura ambiente. El proceso se realizó comenzando con $0.25 \mathrm{~g}$ de quitosana e incrementándose cada vez con $0.25 \mathrm{~g}$ hasta alcanzar $1.25 \mathrm{~g}$, posteriormente a cada tratamiento, las muestras fueron centrifugadas y filtradas. A cada una de la muestras así tratadas se les determinó el color ICUMSA para obtener el porcentaje de color removido respecto a la muestra inicial del jugo. Se realizó una curva de cantidad de quitosana añadido contra porcentaje de color removido.

\section{Pruebas preliminares de remoción de color en los jugos de caña con quitina $y$ quitosana de exoesqueletos y cefalotórax de camarón}

Se realizaron estudios previos para analizar los efectos del tiempo de contacto, la cantidad de agente coagulante, la temperatura y el valor de $\mathrm{pH}$, con la finalidad de encontrar las condiciones más favorables para la mayor remoción de color de los jugos de caña.

\section{Efecto del tiempo de contacto}

Fueron tomadas, por separado, muestras de $60 \mathrm{~mL}$ de cada uno de los jugos las cuales se pusieron en contacto con un gramo de quitosana bajo las siguientes condiciones: agitación de 200 rpm y temperatura ambiente por diferentes periodos de contacto comenzando en 20 min e incrementándose cada vez en 20 min hasta llegar a $80 \mathrm{~min}$. Al término del periodo las muestras fueron centrifugadas y filtradas. A cada una de la muestras así tratadas se les determinó el color ICUMSA para obtener el porcentaje de color removido respecto a la muestra inicial del jugo.

\section{Efecto de la temperatura}

Se llevó a cabo en base a una muestra de $60 \mathrm{~mL}$ de jugo (claro o guarapo), la cual fue puesta en contacto con $\mathrm{lg}$ de quitosana bajo las siguientes condiciones; agitación 200 rpm, a diferentes temperaturas comenzando con $25-28^{\circ} \mathrm{C}$ a incrementos de $5^{\circ} \mathrm{C}$ hasta llegar a $40^{\circ} \mathrm{C}$. Al finalizar el proceso a cada uno de los productos resultantes se les determinó el nivel de color remanente en unidades ICUMSA. 


\section{Remoción de color en los jugos de caña utilizando quitina y quitosana}

Una vez establecidas las condiciones de operación para la remoción de color en los jugos de caña, utilizando quitosana como agente coagulante-floculante, se realizaron las pruebas de remoción de color para cada uno de los jugos. Los ensayos se efectuaron bajo las siguientes condiciones; $60 \mathrm{~mL}$ de muestra de jugo, $200 \mathrm{rpm}$ a temperatura ambiente por un periodo de $60 \mathrm{~min}$. Los productos resultantes fueron sometidos a determinaciones fisicoquímicas de ${ }^{\circ} \mathrm{Brix}, \mathrm{pH}$ y color.

\section{Resultados y discusión}

\section{Caracterización fisicoquímica de los jugos de caña}

Debido a que el jugo de primera extracción es de baja pureza en su composición y aun se encuentra mezclado con los residuos de la molienda era de esperar que la densidad, los sólidos disueltos y color, tuvieran valores mayores que los correspondientes del jugo claro. La Tabla 2 presenta los resultados del análisis de estas características realizadas a ambas muestras de jugo de caña.

Tabla 2. Características fisicoquímicas de los jugos de caña*

\begin{tabular}{|l|c|c|c|c|c|}
\hline \multicolumn{1}{|c|}{ Jugo } & Densidad (g/mL) & ${ }^{\circ}$ Brix & pH & Color (UI) & Sacarosa (g/L) \\
\hline Guarapo & 1.05 & 17.1 & 4.30 & 35333 & 108.9 \\
\hline Claro & 1.04 & 13.5 & 6.27 & 19938 & 121.8 \\
\hline
\end{tabular}

* Valores promedio de dos determinaciones

\section{Caracterización fisicoquímica de los cefalotórax y exoesqueletos de camarón pulverizado}

El exoesqueleto limpio, deshidratado y pulverizado del camarón tuvo $10 \%$ de humedad, $20.9 \%$ de cenizas y densidad de $0.44 \mathrm{~g} / \mathrm{mL}$.

\section{Aislamiento, identificación y características de la quitina de las cabezas y cáscara de camarón}

El procedimiento de aislamiento de la quitina ensayado produjo un material con una coloración beige y $\mathrm{pH}$ neutro. La apariencia física de esta quitina fue muy cercana a la de la quitina comercial. El rendimiento global logrado fue de $50 \%$ (b.s.) de quitina con respecto a la masa de los exoesqueletos empleados.

La Figura 2 presenta los espectros IR que muestran su estrecha semejanza y coincidencia en cuanto a la ubicación e intensidad de las bandas características de los grupos funcionales que permiten confirmar que el material obtenido es quitina.
La quitina obtenida y la comercial fueron caracterizadas de un modo paralelo en términos de humedad: 7.0 y $4.0 \%$; materia insoluble, 62.0 y $80 \%$; cenizas, 13.9 y $10.0 \%$; densidad aparente, 0.3323 y 0.3033 $\mathrm{g} / \mathrm{mL}$, respectivamente. De esta manera se aprecia el grado relativo de pureza del producto obtenido. Es posible observar que los porcentajes de residuos de ignición son un poco más elevados en la quitina aislada en el laboratorio y esto se explica por los posibles restos de impurezas remanentes en ella.

\section{Obtención, identificación y característi- cas fisicoquímicas de la quitosana ob- tenida de exoesqueletos y cefalotórax de camarón}

La quitina recuperada fue sometida a un proceso de desacetilación para ser transformada en quitosana. El material obtenido fue de color blanquecino y de aspecto más brillante que la quitina, con un valor de $\mathrm{pH}$ neutro. El rendimiento logrado para este compuesto fue de $30 \%$ (b.s.) respecto a la masa de los residuos empleados. Este valor es cercano al obtenido por otros estudios como, por ejemplo, Duarte y col. (2009). 


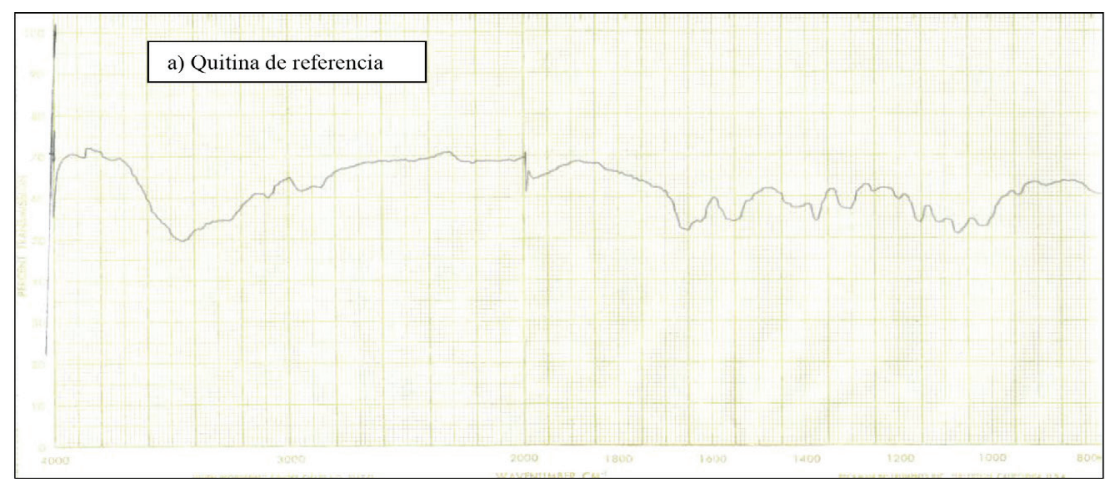

Figura 2a. Espectro infrarrojo de quitina patrón Sigma Chemical Co.

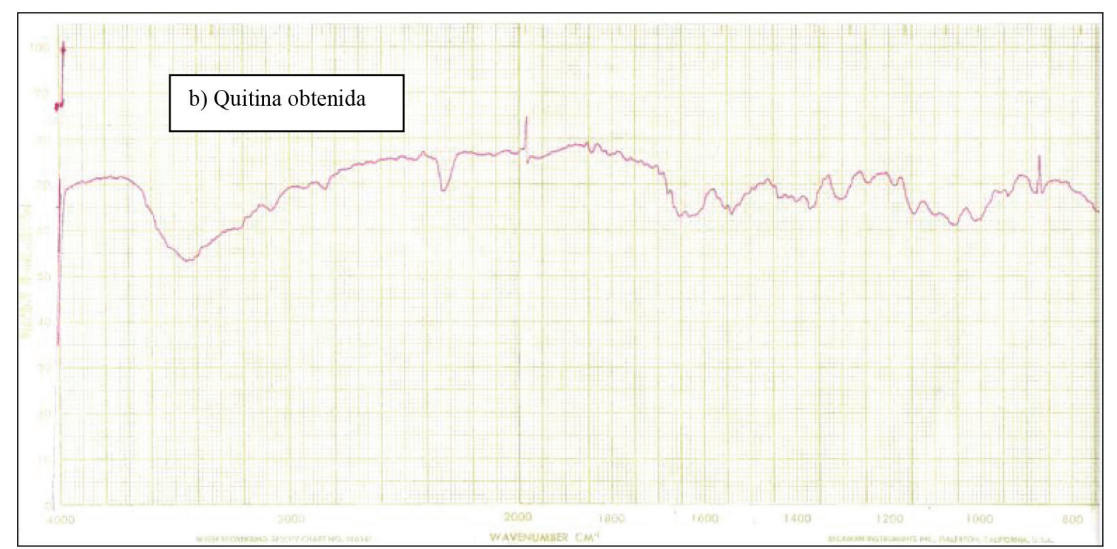

Figura 2b. Espectro infrarrojo de quitina aislada de los exoesqueletos del camarón

El espectro infrarrojo de la quitosana obtenido se muestra en la Figura 3 y fue comparado con un espectro de quitosana patrón Sigma Chemical Co., publicado por Mármol y col. (2004), encontrándose una cercana semejanza en relación con las bandas que lo caracterizan.

La caracterización de la quitosana elaborada en términos de humedad, cenizas, densidad aparente, porcen- taje de materia insoluble, y grado de desacetilación se presenta en la Tabla 3. La comparación de estas características con algunas reportadas en la literatura muestra cercanía a las de la quitosana obtenida. Hernández-Cocoletzi y col. (2009), por ejemplo, prepararon quitosana con GD de $64 \%$ que tuvo una humedad de $10.48 \%$, materia insoluble de $1.8 \%$ y cenizas de $1.4 \%$.

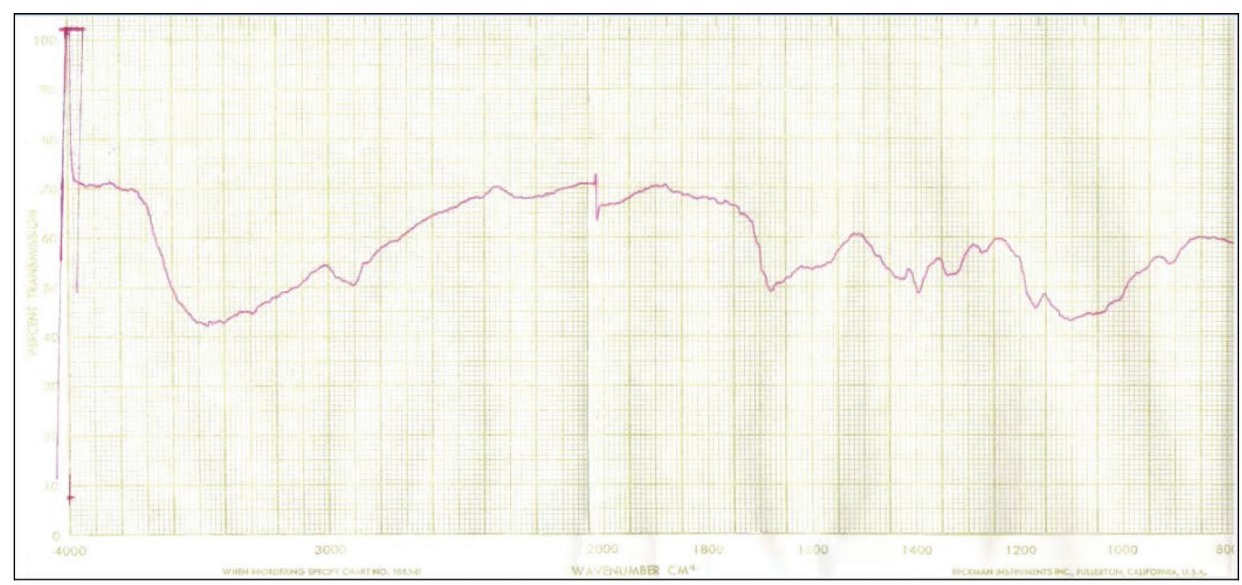

Figura 3. Espectro IR de la quitosana elaborado a partir de exoesqueletos de camarón 
Tabla 3. Caracterización fisicoquímica de la quitosana obtenida*

\begin{tabular}{|l|c|}
\hline Humedad, \% & 9.9 \\
\hline Materia insoluble, \% & 1.22 \\
\hline Cenizas, \% & 1.7 \\
\hline Densidad aparente, $\mathrm{g} / \mathrm{mL}$ & 0.3106 \\
\hline Grado de desacetilación, \% & 64.4 \\
\hline
\end{tabular}

*Valores promedio de dos determinaciones

\section{Ensayos preliminares de remoción de color}

Las pruebas previas de remoción de color se realizaron con la finalidad de observar las condiciones que permitieran los mayores niveles de remoción de color de la quitosana en los jugos de caña. El jugo de caña utilizado fue jugo claro con un nivel de coloración promedio en unidades ICUMSA de 13767. Debido al efecto del $\mathrm{pH}$ en la solubilidad de la quitosana y la quitina se realizaron pruebas que, para el caso de la quitosana, mostraron la necesidad de utilizar un $\mathrm{pH}$ ligeramente ácido y evaluar su rendimiento como agente clarificante del jugo. Los resultados mostraron que, a un valor de $\mathrm{pH}$ de 6.4 y tamaño de partícula de 100 mesh se logró una remoción de color de 67.5 $\%$, respecto al color inicial del jugo.

En el caso de la quitina las pruebas fueron realizadas en condiciones de igualdad a las efectuadas con la quitina comercial, dando como resultado en estos ensayos preliminares una capacidad de remoción de color de la quitina obtenida en el laboratorio de $53.0 \%$, mientras que con la quitina comercial este valor fue de $48.6 \%$.

\section{Remoción de color en jugos de caña de primera extracción y claro}

\section{Efecto del tiempo de contacto}

El periodo en el que se lleva a cabo la operación resulta de suma importancia, ya que periodos largos pueden ocasionar la fragmentación de los flóculos que se forman en el proceso de coagulación, sin embargo, periodos muy cortos provocan que la remoción esperada sea pobre (Nieto-Orellana y Orellana-Ulloa,
$2011)$. Las pruebas realizadas fueron a tiempos de contacto entre las fases de 20, 40, 60 y $80 \mathrm{~min}$. La Figura 4a muestra el efecto del tiempo de contacto en el porcentaje de color removido en el jugo de caña tratado con quitosana. Con base en estos resultados se consideró que el tiempo de contacto adecuado para la evaluación global sería de 60 min por la cercanía al equilibrio mostrada por la tendencia de las curvas.

\section{Efecto de la cantidad de agente coagulante}

Por lo general cuanto mayor es la cantidad de agente clarificante mayor es la cantidad de color removido; sin embargo, como se muestra en la Figura $4 b$, dicha relación no es necesariamente lineal. La Figura $4 \mathrm{~b}$ presenta la tendencia de esta relación para el tratamiento del guarapo y el jugo claro con quitosana. La diferencia entre la remoción alcanzada con 1 y 1.25 $g$ es de aproximadamente $2 \%$ en el guarapo y de $5 \%$ en el jugo claro y la diferencia entre 1 y $0.75 \mathrm{~g}$ es un tanto mayor para el jugo claro de aproximadamente $8 \%$ como se muestra en la Figura $4 \mathrm{~b}$, es por ello que la cantidad de clarificante utilizada en los ensayos posteriores se estableció en $1 \mathrm{~g}$ de quitosana o quitina por cada $60 \mathrm{~mL}$ de jugo.

\section{Efecto de la temperatura}

Se realizaron pruebas con variación de temperatura iniciando a temperatura ambiente, aproximadamente de 25 a $28^{\circ} \mathrm{C}$, por periodos prolongados de una hora, para ambos tipos de jugo. Transcurrido este tiempo se cuantificó el porcentaje de color removido. Los resultado de la Figura $4 \mathrm{c}$ muestran que las remociones de color alrededor de $30^{\circ} \mathrm{C}$ fueron mayores que las que se lograron a temperatura ambiente, para ambos jugos; este efecto fue más notorio para el guarapo; sin embargo, a temperaturas mayores a $40^{\circ} \mathrm{C}$ ésta comenzó a declinar. El jugo claro mostró menores remociones de color después de los $30^{\circ} \mathrm{C}$ y experimentó un proceso de oscurecimiento por efecto de la temperatura como resultado de diversas reacciones bioquímicas de oscurecimiento no enzimático entre algunos de los componentes de los jugos, precursores de color tales como compuestos fenólicos, aminoácidos y glúcidos o azúcares reductores (Paton, 1992), lo que recomendó la realización de los tratamientos de remoción a temperatura ambiente. 


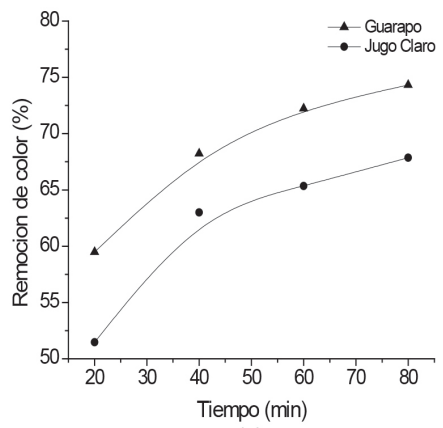

(a)

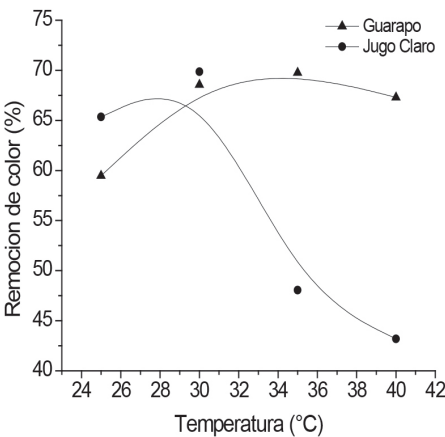

(c)

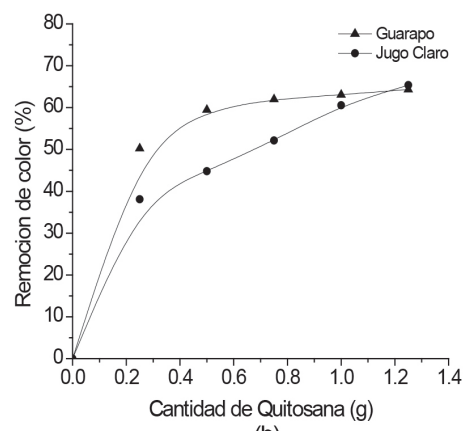

(b)

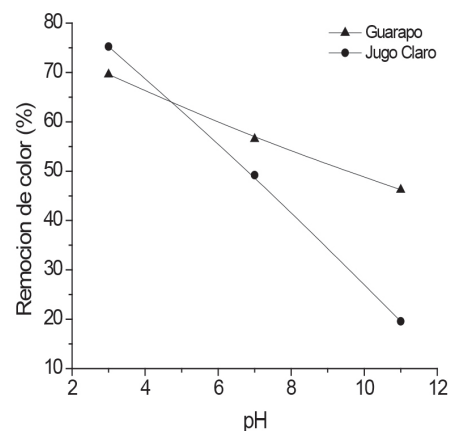

(d)

Figura 4. Efecto del: a) Tiempo de contacto, b) Cantidad de quitosana, c) Temperatura del tratamiento y d) pH del jugo en la remoción de color del jugo de primera extracción (guarapo) y del jugo claro de caña de azúcar tratados con quitosana

\section{Remoción del color de los jugos de caña con quitina y quitosana a diferen- tes valores de $\mathrm{pH}$}

La Figura 4d muestra los porcentajes de remoción de color en función del valor de $\mathrm{pH}$ en ambos tipos de jugo de caña, estudiados. Se hace evidente la tendencia decreciente de esos porcentajes con el aumento del $\mathrm{pH}$ del jugo. La Tabla 4 presenta los resultados de la evaluación de la quitina y quitosana elaboradas a partir de los exoesqueletos de camarón, como agentes removedores de compuestos cromóforos en el jugo de primera extracción o guarapo. La quitina logró clarificar el guarapo desde un valor inicial de $35333 \mathrm{UI}$ hasta otro, al término del tratamiento, de $17699 \mathrm{UI}$ esto a un valor de $\mathrm{pH}$ de 3.0. El comportamiento de esta capacidad de remoción (49.9\%) fue disminuyendo a medida que el tratamiento con quitina se realizó a valores de $\mathrm{pH}$ mayores como cuando se efectuó al valor original del jugo de primera extracción $(\mathrm{pH}=4.30)$ lográndose remover el $44.41 \%$ del color original del jugo. Esta última remoción de color es equivalente a la que representó el proceso de defecación logrado en el ingenio azucarero donde se obtiene jugo claro, o clarificado, de 19938 UI de color.

Tabla 4. Efectos de la quitina y quitosana en la remoción de color en el jugo de primera extracción de caña de azúcar

\begin{tabular}{|l|c|c|c|c|c|c|}
\hline \multirow{2}{*}{$\begin{array}{c}\text { Valor de } \\
\text { pH del } \\
\text { jugo }\end{array}$} & \multicolumn{3}{|c|}{ Tratamiento con quitina } & \multicolumn{3}{c|}{ Tratamiento con quitosana } \\
\cline { 2 - 7 } & ${ }^{{ }^{\circ} \text { Brix }^{\mathbf{a}}}$ & Color $^{\mathbf{b}}$ & Remoción $^{\mathbf{c}}$ & ${ }^{{ }^{\circ} \text { Brix }^{\mathbf{a}}}$ & Color $^{\mathbf{b}}$ & Remoción $^{\mathbf{c}}$ \\
\hline 3.00 & 17.0 & 17699 & 49.90 & 16.6 & 10735 & 69.61 \\
\hline 4.30 & 17.0 & 19640 & 44.41 & 16.8 & 11602 & 67.16 \\
\hline 7.00 & 16.8 & 20448 & 42.12 & 16.6 & 15357 & 56.53 \\
\hline 11.00 & 16.2 & 20508 & 41.95 & 16.4 & 18990 & 46.25 \\
\hline
\end{tabular}

a Al final del tratamiento (valor inicial: $17.1^{\circ} \mathrm{Brix}$ ); ${ }^{\mathrm{b}} \mathrm{Al}$ final del tratamiento en UI (valor inicial: $\left.35333 \mathrm{UI}\right) ;{ }^{c} \mathrm{En} \%$ 
El tratamiento con quitosana, por su parte, representó un $69.61 \%$ de remoción de color del guarapo logrado a un valor de $\mathrm{pH}$ de 3.0 y al igual que en el caso de la quitina los resultados mostraron que la capacidad de remoción de la quitosana disminuyó conforme el pH del jugo se eleva siendo de solamente $46.25 \%$ a pH de 11.0. Es de observarse que el tratamiento con este coagulante-floculante provocó en el jugo tratado una ligera disminución del porcentaje de sólidos solubles presumiblemente por efecto del proceso complejo de coagulación, floculación y adsorción sucedido durante el tratamiento.

En el caso de la quitosana como agente clarificante del jugo de primera extracción superó, en términos del color final del jugo tratado, al logrado mediante defecación (tratamiento químico-térmico) en el ingenio azucarero.
La Tabla 5 presenta los resultados del tratamiento de muestras de jugo claro con quitina y quitosana en términos de la remoción del color de cada uno de ellos. Para este caso el proceso de clarificación fue favorecido también a valores ácidos de pH; a 3.0 el tratamiento con quitina produjo un jugo con un nivel de coloración de 8698 UI lo que representó un $43.6 \%$ del color de las muestras de jugo claro colectadas en el ingenio.

El procesamiento con la quitosana logró remover más compuestos responsables del color del jugo; a pH de 3.0 el jugo tratado con este agente de clarificación tuvo un color de $5140 \mathrm{UI}$ ( $25.7 \%$ de color original de la muestra procesada).

Como puede observarse en la Tabla 5, el tratamiento con quitosana provocó también una leve disminución de los grados Brix respecto al valor inicial estimado en el jugo claro.

Tabla 5. Efectos de la quitina y quitosana en la remoción de color en el jugo claro de caña de azúcar

\begin{tabular}{|l|c|c|c|c|c|c|}
\hline \multirow{2}{*}{$\begin{array}{c}\text { Valor de } \\
\text { pH del } \\
\text { jugo }\end{array}$} & \multicolumn{3}{|c|}{ Tratamiento con quitina } & \multicolumn{3}{c|}{ Tratamiento con quitosana } \\
\cline { 2 - 7 } & ${ }^{{ }^{\circ} \text { Brix }^{\mathbf{a}}}$ & Colorb $^{\text {b }}$ & Remoción $^{\mathbf{c}}$ & ${ }^{{ }^{\circ} \text { Brix }^{\mathbf{a}}}$ & Colorb $^{\mathbf{b}}$ & Remoción $^{\mathbf{c}}$ \\
\hline 3.00 & 13.5 & 8698 & 56.37 & 12.8 & 5140 & 74.22 \\
\hline 6.27 & 13.5 & 9395 & 52.88 & 13.1 & 6908 & 65.35 \\
\hline 7.00 & 13.1 & 10688 & 46.39 & 13.4 & 10128 & 49.20 \\
\hline 11.00 & 12.6 & 17024 & 14.61 & 13.4 & 16036 & 19.57 \\
\hline
\end{tabular}

${ }^{a}$ Al final del tratamiento (valor inicial: 13.5 Brix); ${ }^{b} \mathrm{Al}$ final del tratamiento en UI (valor inicial: 19938 UI); ${ }^{c} \mathrm{En} \%$

Finalmente, en cuanto a la remoción del color del guarapo con quitina, se encontró mayor remoción [estadísticamente significativa $\mathrm{P}<0.05$ ] con un $\mathrm{pH}$ de 3 que con todos los demás.

\section{Conclusiones}

Los resultados mostraron que el guarapo y el jugo clarificado provenientes de un ingenio azucarero tuvieron originalmente niveles de color de 35333 y 19938 UI, respectivamente y que la quitina y la quitosana obtenidos de residuos de camarón lograron remover hasta un 49.9 y $69.61 \%$, respectivamente, del color del jugo de primera extracción; y 56.37 y $75.22 \%$, respectivamente, del color original del jugo claro de caña en tratamientos realizados a un $\mathrm{pH}$ de 3.0 y temperatura ambiente. Ambos materiales mostraron potencialidad como alternativa de purificación del jugo de caña de azúcar.

\section{Reconocimientos}

Los autores agradecen al personal del ingenio cooperante y especialmente al Ing. Manuel Enríquez-Poy de la Cámara Nacional de las Industrias Azucarera y Alcoholera de México por su valioso apoyo para este proyecto. Asimismo, agradecen los valiosos comentarios del Dr. Ronny Flores al manuscrito. 


\section{Referencias}

Aguilar-Rivera, N. A., Galindo, M. G., Fortanelli, M. J. y Contreras, S. C. 2009. ¿Por qué diversificar la agroindustria azucarera en México?. GCG: Revista de Globalización, Competitividad y Gobernabilidad, 3(1):62-75.

Beléndez-Moreno, L. F. J., Espino-Barr, E., Galindo-Cortes, G., Gaspar-Dillanes, M. T., Huidobro-Campos, L. y Morales-Bojórquez, E. Editores. 2014. Sustentabilidad y Pesca Responsable en México. Evaluación y Manejo. Dirección electrónica: http://www.inapesca.gob.mx/portal/documentos/publicaciones/LIBROS/Sustentabilidad-y-Pesca-Responsable-en-Mexico.-2014-web.pdf

Bough, W. A. 1975. Reduction of suspended solids in vegetable canning waste effluents by coagulation with chitosan. Journal of Food Science. 40(2):297-301.

Bough, W. A. y Landes, D. 1978. Recovery and nutritional evaluation of proteinaceous solids separated from whey by coagulation with chitosan. Journal of Dairy Science. 59(11):1874-1880.

Cañipa-Morales, A. J. 1994. Obtención de quitina a partir de cefalotórax de camarón. Tesis de Maestría en Ciencias de los Alimentos (Química). Facultad de Química, UNAM. Noviembre 29. México D.F., México.

Castro-Domingues, R. C., Braz-Faria Jr., S., Bernardes-Silva, R., Luiz-Cardoso, V., Miranda-Reis, M. H. 2012. Clarification of passion fruit juice with chitosan: Effects of coagulation process variables and comparison with centrifugation and enzymatic treatments. Process Biochemistry. 47(3):467-471.

Chen J. C. P. 1991. Manual del azúcar de caña para fabricantes de azúcar de caña y químicos especializados. Limusa, México, D.F., México

Clarke, M. A., Roberts, E. J. 1996. Removal of color polysaccharides, phenolics, and turbidity from sugar-containing solutions and derivated fibrous residues therefore. Pat 5504196. Washington, D.C. EE.UU.

Clarke, M. A. y Godshall, M. A. Editores. 2013. Chemistry and processing of sugarbeet and sugarcane. Proceedings of the Symposium on the Chemistry and Processing of Sugarbeet, Denver, Colorado, April 6, 1987, and the Symposium on the Chemistry and Processing of Sugarcane, New Orleans, Louisiana, September 3-4, 1987. Sugar Series. Vol. 9. Elsevier Science, Adobe PDF eBook (33 Mb). Dirección electrónica: https://www.overdrive.com/media/2016025/chemistry-and-processing-of-sugarbeet-and-sugarcane

COLPOS. 1991. Industria azucarera. Determinación de pol (sacarosa aparente) en muestras de jugos de especies vegetales productoras de azúcar. Dirección electrónica: http://www.colpos.mx/bancodenormas/nmexicanas/NMX-F-271-1991.PDF

DOF. 1991. Norma Mexicana NMX-F-271-1991. INDUSTRIA AZUCARERA. Determinación de pol (sacarosa aparente) en muestras de jugos de especies vegetales productoras de azúcar. método del peso normal. Sugar industry. Determination of pol (apparent saccharose) in juice samples of vegetal species which produce sugar - standard weight method. Normas Mexicanas. Dirección General de Normas. Diario Oficial de la Federación. Estados Unidos Mexicanos.

Domínguez, L. 2005. Desarrollo regional y competitividad: La agroindustria azucarera en México. Nóesis. 15(27):227-250.

Duarte, E., Verbel, J. O., Jaramillo, B. E. (2009). Remoción de cromo de aguas residuales de curtiembres usando quitosan obtenido de desechos de camarón. Scientia et Technica, 2(42):290-295.

Enríquez-Poy, M. 2015. Conferencia inaugural. En Taller Internacional de Expertos sobre Manejo de Malezas, Maduradores, Bio-Estimulantes y Productos Bioactivos Foliares en el Cultivo de la Caña de Azúcar. Enero 28-30. Peñuela, Veracruz, México. ATAM. 28(1):5-8.

Flores-Ortega, R. A. 2004. Bioplástico de quitina: Formación de películas de quitina a partir de desechos de camarón por métodos ecológicos. Tesis de Maestría en Ciencias. Programa de Maestría y Doctorado en Ciencias Químicas, UNAM, Facultad de Química. México, D. F. México.

Flores-Ortega, R.A. 2008. Obtención y caracterización de esponja de quitina a partir de cefalotórax de camarón. Tesis de Doctorado en Ciencias. Programa de Maestría y Doctorado en Ciencias Químicas, UNAM, Facultad de Química. México, D. F. México.

Flores-Ortega, R., Barrera-Rodríguez, S., Durán-Domínguez-de-Bazúa, C. 2004. Extracción ecológica de quitina y subproductos. Solicitud de Registro de Patente: Octubre 1, 2004. Facultad de Química, UNAM. IMPI. PA/a/2004/009517. Patente Núm. 264482. Otorgada el 12 de febrero de 2009. México D.F., México.

Goycoolea, F., Argüelles, W., Peniche, C., Higuera, I. 2000. En Novel macromolecules in food systems. Series Developments in Food Science. Vol. 41. G. Doxastakis y V. Kiosseoglou. Pp. 265-307. Elsevier Applied Science. Amsterdam, Países Bajos.

Hernández-Cocoletzi, H., Águila-Almanza, E., Flores-Agustín, E. L., Viveros-Nava E. y Ramos-Cassellis, E. 2009. Obtención y caracterización de quitosana a partir de exoesqueletos de camarón. Superficies y Vacío. 22 (3): 57-60.

Hildalgo, C., Fernández, M., Nieto, O., Paneque, A., Fernández, G. y Llópiz, C. 2009. Estudios de quitosanas cubanos derivados de la quitina de la langosta. Revista Iberoamericana de Polímeros 10 (1): 11-27

Hinkova, A., Bubnik, Z., Kadlec, P. y Pridal, J. 2002. Potentials of separation membranes in the sugar industry. Separation and Purification Technology. 26:101-110. 
Honig, P. Ed. 2013. Principles of Sugar Technology. Elsevier Press Inc. Nueva York, NY. EE.UU.

Horwitzs, W. 1995. Official methods of analysis of the Association of Official analytical Chemists. AOAC Washington DC. EEUU

ICUMSA. 2006. ICUMSA Libro de Métodos (2005). Verlag Dr. Albert Bartens KG, Berlín, Alemania.

Lárez-Velásquez, C. 2006. Quitina y quitosano: materiales del pasado para el presente y el futuro. Avances en Química. 1(2):15-21.

Mármol, Z., Cardoso, J., Carrasquero, S., Paez, G., Chandler, C., Araujo, K., Rincón, M. 2009. Evaluación de polifenoles totales en vino blanco tratado con quitina. Revista de la Facultad de Agronomía Universidad del Zulia (LUZ). 26:423-442.

Mármol, Z., Gutiérrez, E., Páez, G., Ferrer, J. y Rincón, M. 2004. Desacetilación termoalcalina de quitina de conchas de camarón. Multiciencias. 4:1-10.

Mármol, Z., Páez, G., Rincón, M., Araujo, K., Aiello, C., Chandler C. y Gutiérrez E. 2011. Quitina y quitosano polímeros amigables. Una revisión de sus aplicaciones. Revista Tecnocientífica URU. 1:53-58.

McKay, G., Blair, H. S. y Grant, S. 1987. Desorption of copper from a copper-chitosan complex. Journal of Chemical Technology and Biotechnology, 40, 63-74

Montgomery, D. C. 2011. Bioestadística aplicada y probabilidad para ingenieros / Applied Statistics and Probability for Engineers. $3^{a}$ Ed. Dirección electrónica: http://www.upch.edu.pe/facien/fc/dbmbqf/zimic/ubioinfo/bks/Bioestadistica/applied\%20statistics\%20and\%20 probability\%20for\%20engineers\%20-\%20montgomery.pdf

Nieto-Orellana, C. R. y Orellana-Ulloa, V. P. 2011. Aplicación del quitosano como promotor de floculación para disminuir la carga contaminante. Tesis Doctoral. Universidad Politécnica Salesiana. Cuenca, Ecuador. 132 pag.

Oszmia ski, J. y Wojdyło, A. 2007. Effects of various clarification treatments on phenolic compounds and color of apple juice. European Food Research and Technology. 224(6):755-762.

Ortega-Granados, J. A. 2014. Caracterización mecánica de productos espumados obtenidos de residuos sólidos de las pesquerías para su posible aplicación en la ingeniería ambiental. Tesis de Maestro en Ingeniería, UNAM. México D.F. México.

Ortega-Granados, J. A. y Durán-Domínguez-de-Bazúa, M. C. 2014. Proceso de química verde para la obtención de materiales espumados a partir de residuos sólidos de animales artrópodos, y productos obtenidos con el mismo. Solicitud de Registro: Diciembre 10, 2014. Facultad de Química, UNAM. Coordinación de Gestión de Propiedad Intelectual, Coordinación de Propiedad Intelectual, Dirección General de Vinculación, Coordinación de Innovación y Desarrollo de la UNAM. Solicitud de registro con el IMPI expediente MX/a/2014/015119 folio MX/E/2014/088656. Dirección General de Invenciones y Marcas. Departamento de Patentes. Secretaría de Patrimonio y Fomento Industrial. México D.F., México.

Paananen, H. A. y Wen, T. 1999. Process for decolorization of solutions. Pat 5932016. Washington, D.C. EEUU.

Paton, N. H. 1992. The origin of colour in raw sugar. En: Proc. Australian Society of Sugar Cane Technol. Conference, 14. pp. 8-17.

Qudsieh, H. Y. M., Yusof, S., Osman, A., Rahman, R. A. 2002. Effect of maturity on chlorophyll, tannin, color, and polyphenol oxidase (PPO) activity of sugarcane juice (Saccharum officinarum Var. Yellow Cane). Journal of Agricultural and Food Chemistry. 50(6):1615-1618.

RAE. 2015. Diccionario de la lengua española (DRAE), obra de referencia de la Academia. Última edición, 23. ${ }^{\text {a }}$, publicada en octubre de 2014. Dirección electrónica: http://lema.rae.es/drae/?val=guarapo

Šereš, Z., Gyura, J., Eszterle, M. y Djuri, M. 2006. Separation of non-sucrose compounds from syrup as a part of the sugar-beet production process by ultrafiltration with ceramic membranes. European Food Research and Technology. 223(6):829-835.

Statgraphics Centurion XV:II. Dirección electrónica: http://www.statgraphics.net/descargas/

Tastan, O. y Baysal, T. 2015. Clarification of pomegranate juice with chitosan: changes on quality characteristics during storage. Food Chemistry. Aug 1;180:211-8. doi: 10.1016/j.foodchem.2015.02.053. Epub 2015 Feb 16.

Téllez-Castañeda, M. 2014. Una industria con gran futuro... cultivo de camarón (II). Dirección electrónica: http://eleconomista.com.mx/ columnas/agro-negocios/2014/10/23/industria-gran-futuro-cultivo-camaron-ii

Tolaimate, A., Desbrieres, J., Rhazi, M., Alagui, A., Vincendon, M., Vottero, P. 2000. On the influence of deacetylation process on the physicochemical characteristics of chitosan from squid chitin. Polymer. 41(7):2463-2469. 